\title{
Letter to the editor. Spotlight on measles 2010: Measles in healthcare workers - vaccination should be revisited
}

E Botelho-Nevers ${ }^{1}$, L Chevereau ${ }^{1}$, P Brouqui (philippe.brouqui@univmed.fr) ${ }^{1}$

1. Pôle de Maladies Infectieuses, Hôpital Nord, Assistance Publique-Hôpitaux de Marseille, France

Citation style for this article:

Botelho-Nevers E, Chevereau L, Brouqui P. Letter to the editor. Spotlight on measles 2010: Measles in healthcare workers - vaccination should be revisited.

Euro Surveill. 2010;15(41):pii=19687. Available online: http://www.eurosurveillance.org/ViewArticle.aspx?Articleld=19687

Article published on 14 October 2010

To the editor: In the light of the current outbreak of measles in France reported by Parent du Chatelet et al. [1], we would like to report a case of hospital-acquired measles in a nurse who had not received the measlesmumps-rubella (MMR) vaccine. Working in our department of infectious diseases, she was infected in spite of barrier measures.

On 8 August 2010 a woman in her 20 s was admitted to our department with a maculopapular rash associated with high-grade fever and cough. The cause was rapidly laboratory-confirmed as measles. From the moment of her admission in our ward to her discharge on 13 August she was confined to a single room and respiratory isolation measures were in place. As of 9 August, a nurse in her 20 s took care of the patient using protective personal equipment including an FFP2 facial mask and alcohol-based hand rub.

Thirteen days after the first contact with the patient, the nurse presented with fever and four days later developed a maculopapular rash. She was laboratoryconfirmed with measles which was complicated by keratitis. Following a 15-day sick leave the nurse recovered. She had had no contact with a case of measles in the community. A survey of other members of staff and patients in contact with the nurse was carried out. No other secondary cases of measles were described. One medical student without immunity to measles was vaccinated. It was not possible to establish a molecular link between the viruses in our two cases as all the measles virus genotypes circulating during the current local outbreak were identical.

The case reported here is noteworthy because an unvaccinated nurse trained in infectious diseases contracted measles in spite of efficient use of respiratory protective measures and alcohol-based hand rub. A recrudescence of measles is currently occurring in France, especially among children and young adults, due to insufficient vaccine coverage in these population groups [1]. Consequently young healthcare workers (HCW) are at risk of occupational measles if they are not immunised. In the literature, nosocomial transmission of measles from HCW to patients and from patients to unimmunised HCW has been reported
$[2,3]$. Indeed measles is a highly contagious disease with a basic reproduction number ranging from 7.7 to 15 [4], as the transmission airborne droplets leads to a high risk of infection for unvaccinated or not naturally immunised individuals even if isolation measures are correctly applied. Vaccination is the only reliable protection against nosocomial spread of measles. Reports of susceptibility to measles showed a high level of immunity, including natural immunity, among HCW in Europe[5]. Therefore, even if the prevalence of nonimmune HCW seems to be low, the low uptake of MMR immunisation and the increase in measles outbreaks [1] may increase the risk of nosocomial transmission. .

It should be mandatory to identify non-immune HCW and offer them vaccination. Only HWC who are vaccinated or willing to be vaccinated should be recruited to work on medical wards, especially high risk wards such as infectious disease, emergency room, paediatric, maternity and oncology wards. This recommendation should also extend to medical students who are often poorly protected against vaccine-preventable diseases as seen in our case.

If mandatory vaccination is not possible in France as we saw during the 2009 influenza pandemic, a strategy of voluntary vaccination for HCW should be rapidly implemented in hospitals, especially in high risk areas and even on infectious disease wards where isolation barriers are usually used carefully.

\section{References}

1. Parent du Châtelet I, Antona D, Freymuth F, Muscat M, Halftermeyer-Zhou F, Maine C, Floret D, Lévy-Bruhl D. Spotlight on measles 2010: Update on the ongoing measles outbreak in France, 2008-2010. Euro Surveill. 2010;15(36): pii=19656. Available from: http://www.eurosurveillance.org/ViewArticle. aspx?Articleld $=19656$

2. Tafuri S, Germinario C, Rollo M, Prato R. Occupational risk from measles in healthcare personnel: a case report. J Occup Health. 2009;51(1):97-9.

3. Grgic-Vitek M, Frelih T, Ucakar V, Prosenc K, Tomažič J, Petrovec M, Kraigher A. Spotlight on measles 2010: A cluster of measles in a hospital setting in Slovenia, March 2010. Euro Surveill. 2010;15(20):pii=19573. Available online: http://www. eurosurveillance.org/ViewArticle.aspx?Articleld=19573

4. Mossong J, Muller CP. Estimation of the basic reproduction number of measles during an outbreak in a partially vaccinated population. Epidemiol Infect. 2000;124(2):273-8.

5. Fedeli U, Zanetti C, Saia B. Susceptibility of healthcare workers to measles, mumps rubella and varicella. J Hosp Infect. 2002;51(2):133-5. 